\title{
Non-mechanical axial motion compensation using Master-Slave optical coherence tomography
}

\author{
Adrian Fernández*, Manuel J. Marques, Adrian Bradu, and Adrian Podoleanu' \\ Applied Optics Group, School of Physical Sciences, University of Kent, Canterbury CT2 7NH, United Kingdom. \\ af498@kent.ac.uk
}

\begin{abstract}
We present a novel technique for accurate, non-mechanical, axial motion compensation for OCT in both ophthalmology and dermal imaging through the combination of MS-OCT for imaging and a LCl for the motion detection. (C) 2020 The Author(s)

OCIS codes: 000.0000, 999.9999.
\end{abstract}

Upon biological imaging examination, the stabilization of the sample to be observed is required in order to acquire a well-resolved image which is free of any motion artefacts. Due to the nature of in-vivo samples, such as the human retina, it is impossible to ensure its complete immobilization, thus being necessary to accurately compensate its inherent motion.

In this article, we propose a new axial motion compensation method based on the properties of Master-Slave Optical Coherence Tomography (MS-OCT). OCT is a non-invasive, contactless imaging technique which allows to examine the depth profile of a sample, A-scan, through the interference between the light returned from the sample and that from a reference arm.

Consecutive A-scans make a cross-section in depth along one lateral coordinate called B-scan. A 3D volume can be obtained scanning in 2 lateral coordinates, and the plane made by every lateral position at a specific depth is called en-face or C-scan [1]. While movements during the acquisition of an A-scan are rare due to the speed of the acquisition of an A-scan, between $70 \mathrm{kHz}$ and $100 \mathrm{kHz}$ in most commercial systems, motion artefacts can be an issue for B-scans and C-scans.

Most of the approaches to this problem can be categorised as mechanical or software-based solutions. Mechanical-based systems usually employ an extra acquisition mechanism which will feed the axial position that will be translated into a mechanical displacement inside the reference arm of the imaging interferometer to change its optical path difference (OPD) [2]. Software-based systems tend to follow a model of the movement or to analyse the changes between two acquisitions to decode the displacement and compensate it in the images acquired [3].

While mechanical systems are more accurate than software-based systems, they tend to be more expensive and have increased latency due to the physical motion of the components. Frequency-domain OCT (FD-OCT) methods typically require a Fast Fourier Transform (FFT) of the channeled spectra acquired in the interferometer to decode the A-scan. On the other hand, the OCT method we have introduced, Master-Slave (MS), does not require a FFT for the same A-scan decoding operation [4]. In a first stage called Master, the sample is replaced with a mirror and we characterize the system at different OPDs. In this characterization, we store interference spectra, called masks. These contain the non-linearities and dispersion of the system. In a second stage called Slave, the mirror is replaced by the sample under study and the channeled spectra are compared against the masks, thus obtaining A-scans. The OPD range covered is that of the OPD values used to acquire the masks. Therefore, selecting a subset of masks is equivalent to a change in OPD which would otherwise be achieved by mechanically modifying the length of the reference arm, for instance.

In this communication we present an imaging system capable of compensating axial movements of the sample by the joined action of two sub-systems, schematically represented in Fig. 1 (a): a system employed for imaging, referred as imaging sub-system (IS), and another employed for axial tracking, referred as axial tracking subsystem (ATS). The ATS employs an interferometer, illuminated by a $1300 \mathrm{~nm}$ swept source with a $10 \mathrm{kHz}$ sweeping frequency (ATS source, Santec HSL-2000-12-MDL). The ATS sample beam is focused on the cornea, in the case of ophthalmic applications, or on the first surface of the sample to be imaged, in the case of dermal imaging. The resulting modulated spectra is detected by a balanced photo-detector BPD (Santec BPD-200, 200MHz cutoff frequency), and the corresponding electrical signal is digitized into the computer via a National Instruments $\mathrm{PCI} 5124$ card with a $25 \mathrm{MS} / \mathrm{s}$ sampling rate. For the IS, we used a swept source with a $2 \mathrm{kHz}$ sweeping frequency, 
$850 \mathrm{~nm}$ central wavelength and a tuning range of $50 \mathrm{~nm}$ (IS source, Superlum BroadSweeper 840 ). The resulting modulated spectra are detected by a custom-made balanced photo-detector, BPD (1MHz cutoff frequency) and the corresponding electrical signal is digitized via a AlazarTech ATS9350 acquisition card sampling at $2 \mathrm{MS} / \mathrm{s}$. By setting appropriate spectral tuning ranges, both sub-systems have the same axial resolution in air of $6.7 \mu \mathrm{m}$. In order to implement axial motion compensation, the channeled spectra from the ATS are processed using a FFT, and its maximum is used to select the starting point of the subset of masks employed in the IS. The compensation is applied to individual A-scans, allowing it to operate both inter- (Fig. 1 (b1-b2)) and intra-B-scan (Fig. 1 c1-c2).
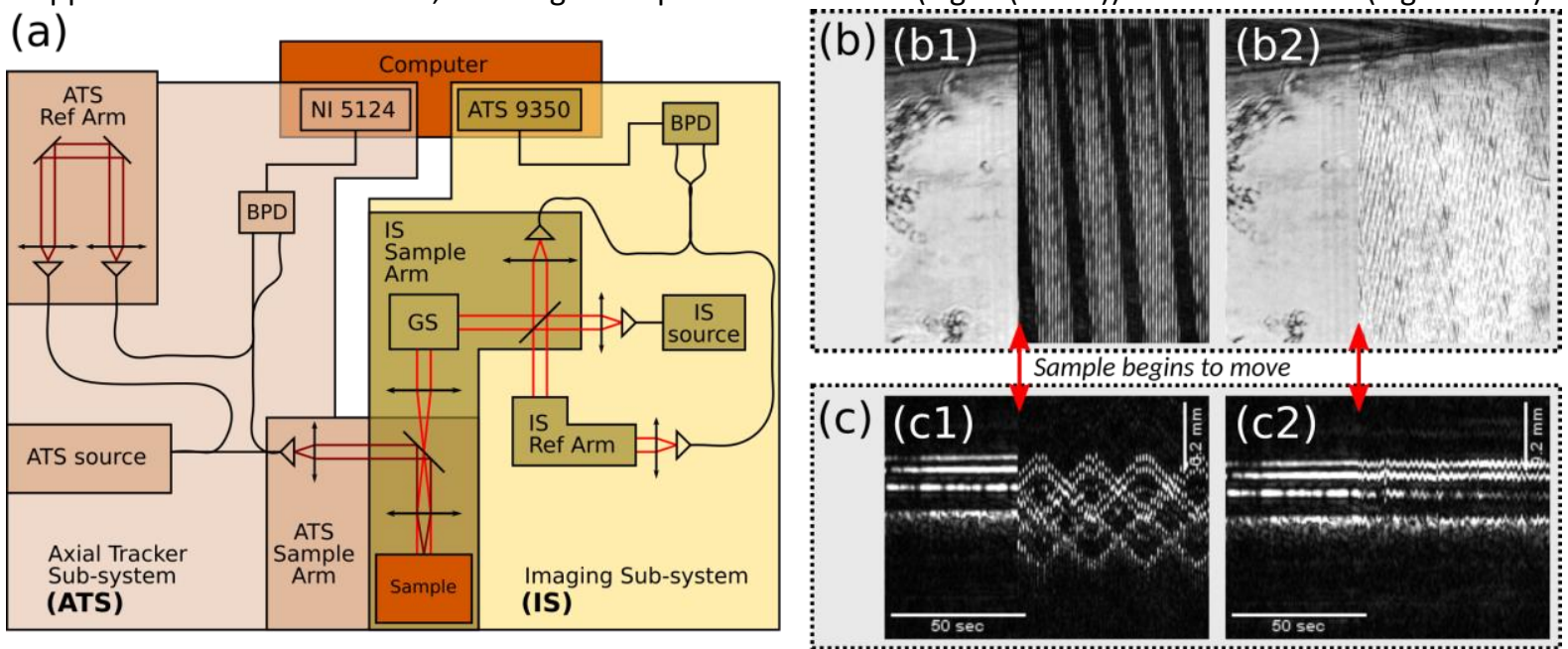

Fig. 1. (a) Schematic representation of the two sub-systems providing axial compensation and imaging. BPD, balanced photo-detector; GS, pair of galvanometer scanners; (b) En-face C-scan images acquired without (b1) and with (b2) axial motion compensation. (c) M-mode images acquired without (c1) and with (c2) axial motion compensation.

We used a moving subset of 150 masks, equivalent to a $1 \mathrm{~mm}$ axial interval measured in air, over a range of 500 masks, equivalent to $3.35 \mathrm{~mm}$, to process each A-scan. Therefore, we compensated axial movements up to $\pm 1.175 \mathrm{~mm}$. To demonstrate the concept, an artificial eye (Rowe Technical Design OCT Model Eye) was mounted on a translation stage (Newport VP-25XA) as a moving sample to be imaged. The stage was controlled with linear motion amplitudes of $0.2 \mathrm{~mm}, 0.5 \mathrm{~mm}$ and $1 \mathrm{~mm}$, and speeds of $0.5 \mathrm{~mm} / \mathrm{s}, 1 \mathrm{~mm} / \mathrm{s}$ and $2 \mathrm{~mm} / \mathrm{s}$. The resulting images were corrected both in real-time and in post-acquisition. With this method, motion artefacts were reduced; to quantify this reduction, the numerical cross-correlation between B-scan frames was computed. Prior to the start of the sample movement, the normalized correlation value between B-scans was $\approx 0.9$ (on average). With the introduction of the sample motion, the cross-correlation value between B-scans drops to $\approx 0.26$ with no correction applied; with the ATS sub-system engaged, this figure increases to $\approx 0.75$. The drop from 0.9 to 0.75 when the ATS sub-system is employed may be attributed to residual lateral vibrations from the stage, since only axial compensation was applied.

Further results, including in-vivo dermal imaging, will be presented in the conference proving the usefulness of the Master Slave principle for non-mechanical axial motion compensation.

References

1. A. Podoleanu, "Optical Coherence Tomography," J. Microsc. 247, 209-219 (2012).

2. M. Pircher, B. Baumann, E. Gotzinger, H. Sattmann, and C. K. Hitzenberger, "Simultaneous SLO/OCT" imaging of the human retina with axial eye motion correction," Opt. Express 15, 16922-16932 (2007).

3. B. Braaf, K. V. Vienola, C. K. Sheehy, Q. Yang, K. A. Vermeer, P. Tiruveedhula, D. W. Arathorn, A. Roorda, and J. F. d. Boer, "Real-time eye motion correction in phase-resolved OCT angiography with tracking SLO," Biomed. Opt. Express 4, 51-65 (2013).

4. S. Rivet, M. Maria, A. Bradu, T. Feuchter, L. Leick, and A. Podoleanu, "Complex master slave interferometry," Opt. Express 24, 2885 (2016). 